\title{
Meat Condemnations and Economic Importance in the Northern and Southern Egyptian Abattoirs
}

\section{Eman Sayed Mohammed ${ }^{1}$, Mohamed Abdelfattah MaKY ${ }^{2 *}$ \\ ${ }^{1}$ Parasitology Department, Faculty of Veterinary Medicine, South Valley University, 83522, Qena, Egypt; ${ }^{2}$ Food Hygiene and Control Department, Faculty of Veterinary Medicine, South Valley University, 83522, Qena, Egypt.}

\begin{abstract}
Food animals are important sources of protein of high quality and essential nutrients. However, a large number of carcasses and offals are condemned in slaughterhouse as a result of various diseases. An abattoir study was performed at two uninvestigated regions in Egypt for two years (2017-2018) in order to identify the chief causes of meat condemnation and the subsequent economic loss. A retrospective survey was carried out on the North and South of Egypt, The northern region was represented by Alexandria abattoir and owing to the full lack of information in the Southern region it was represented by two abattoirs; Qena and Aswan abattoirs. Ante-mortem and postmortem examinations were carried out and the findings of inspections were recorded and analyzed. Moreover, the financial loss was determined. Out of 167812 animals were slaughtered in the three abattoirs, $68(0.04 \%)$ animals were totally condemned. In addition to, 3198 (1.9\%) lungs, 1447 (0.86\%) hearts, 4290 (2.55\%) livers, 535 (0.31\%) heads, $291(0.17 \%)$ kidneys and $765(0.45 \%)$ spleens were condemned. This work revealed that the economic loss due to meat condemnation in three slaughterhouses was 4529010 Egyptian pound (383063 USD). The main causes of meat condemnation were tuberculosis, icterus, parasitic cyst, pneumonia, hydronephrosis and fascioliasis. The incidence of meat rejection and financial loss in the Aswan slaughterhouse was significantly high. The revenue damage caused by meat condemnations was high in comparison to the amount of local income. The findings of the current work highlighted the necessity for the development of an effective control program for the causes of meat condemnation in Egypt.
\end{abstract}

Keywords | Abattoir, Economic loss, Inspection, Meat condemnations

\footnotetext{
Received | August 28, 2019; Accepted | November 14, 2019; Published | January 03, 2020

*Correspondence | Mohamed Abdelfattah Maky, Department of Food Hygiene and Control, Faculty of Veterinary Medicine, South Valley University, 83522, Qena, Egypt; Email: mohamedmekky@vet.svu.edu.eg

Citation | Mohammed ES, Maky MA (2020). Meat condemnations and economic importance in the northern and southern egyptian abattoirs. Adv. Anim. Vet. Sci. 8(1): 96-107.

DOI | http://dx.doi.org/10.17582/journal.aavs/2020/8.1.96.107

ISSN (Online) | 2307-8316; ISSN (Print) | 2309-3331
}

Copyright (C) 2020 Mohammed et al. This is an open access article distributed under the Creative Commons Attribution License, which permits unrestricted use, distribution, and reproduction in any medium, provided the original work is properly cited.

\section{INTRODUCTION}

$\mathrm{T}$ he human community is rapidly multiplying in various countries all over the world. However, the rate of multiplication is above the multiplication rate of food animals, particularly in developing areas (Steinfeld et al., 2006). The produced meat from food animals is the major source of high-quality protein and precious food throughout the world. The chief source of red meat is cattle, camel, sheep and goat. However, certain affections caused by parasites, bacteria and viruses restrict the production of meat (Bin Kabir et al., 2010; Fekadu et al., 2012). These illnesses resulting in huge losses ranging from the morality of livestock, decrease the weight and condemnation of meat at slaughterhouses.

Parasitic diseases have a critical effect on human and livestock in developing nations (Abdulhameed et al., 2018). Cysticercosis, dicrocoeliasis, hydatidosis and fascioliasis are major parasitic diseases that have a considerable effect on human health and the safety of meat (Aminzare et al., 2018; Pezeshki et al., 2018). Bovine Cysticercosis can be transmitted to man through the ingestion of infected meat with Cysticercus bovis, while animals are responsible for completing the life cycle in case of hydatidosis and fascioliases (Youssef and Uga, 2014). Moreover, bovine 
cysticercosis is responsible for the condemnation of the carcass in heavy infected cases. In addition, food animals are able to harbor large number of pathogenic bacteria; these bacteria can be transferred to human during preparation, handling and consumption of infected meat as well as condemnation of meat at slaughterhouse (Fromsa and Jobre, 2012).

Therefore, meat inspection is a useful tool in the detection of animal diseases and in preventing the spread of diseased meat that could harm consumers. Data generated by the inspection of live and slaughtered food animals are valuable epidemiological information for disease assessment and determining the efficacy of the preventive and therapeutic intervention.

Egypt has a great animal population in Africa with approximately 9.5 million heads and 484 abattoirs as reported by the ministry of agriculture and land reclamation in 2019. Slaughterhouses played a crucial role in distinguishing the meat with different affections and thus preventing their marketing to consumers (Alton et al., 2010). There are inadequate data about various illnesscausing meat condemnations and their financial loss in Egyptian abattoirs, particularly in southern Egypt. Hence, the aims of this work were to identify the causes of meat condemnations in three Egyptian abattoirs in 2017 and 2018, one abattoir in northern Egypt (Alexandria), while two abattoirs (Qena and Aswan) represented Southern Egypt. In addition to the financial loss due to meat condemnation was estimated. The findings of this study might contribute to establishing a suitable policy for the banning and monitoring of animal diseases in Egypt particularly in southern Egypt.

\section{MATERIALS AND METHODS}

\section{STUDY AREA}

The study was carried out in three Egyptian slaughterhouses where food animals were slaughtered for local consumption. One in northern Egypt, in the province of Alexandria, and two slaughterhouses in the upper of Egypt, including the province of Qena and Aswan. Alexandria is the second biggest town in Egypt after Cairo, it stretching alongside the Mediterranean Sea and having a population about 5.200.000. Qena is a province in Upper Egypt, located on the east bank of the Nile River, characterized by warm weather and with a population of approximately 230.392. Aswan is situated in southern Egypt and has the hottest summer period with a population around 290.327 (Figure 1).

\section{Study Duration And Animals Population}

The work was conducted over the course of 24 months from January 2017 to December 2018. The slaughtered and inspected animals during the study were 126686, 18886 and 22240 animals in Alexandria, Qena and Aswan abattoirs, respectively. The investigated animals in Qena and Aswan were native cattle, buffalo, camel, sheep and goat. While, the Alexandria abattoir is capable of slaughtering the same species of animals with imported cattle.

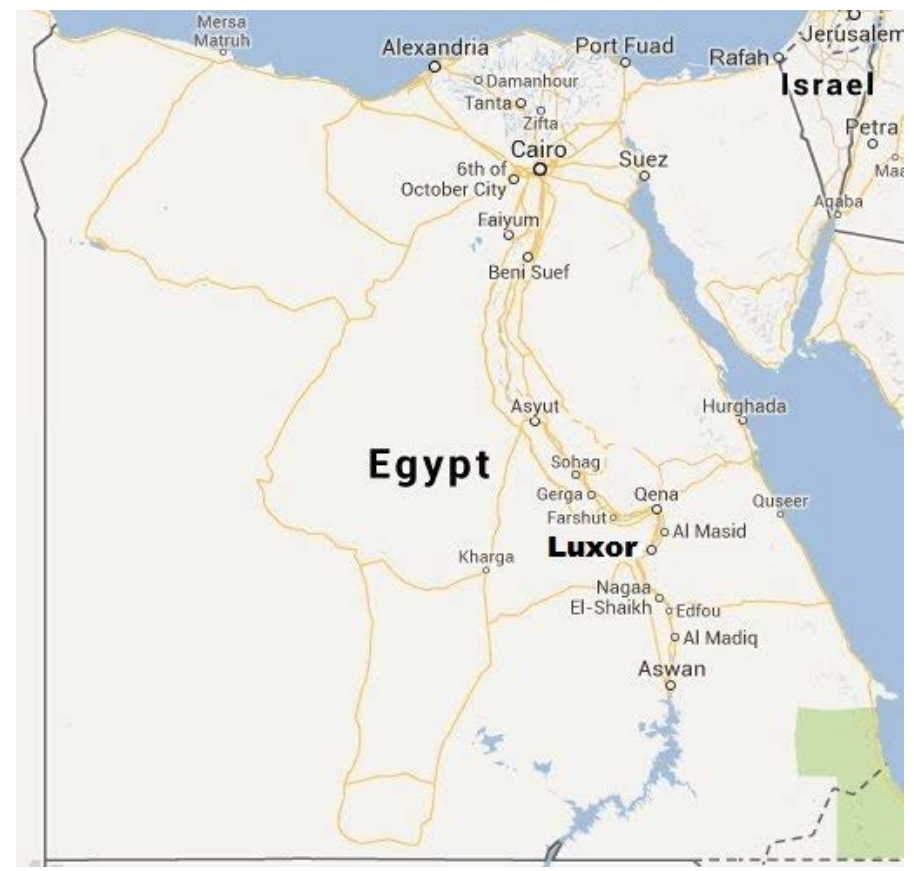

Figure 1: Locations of the study in Egypt were indicated by Blake circle. Locations of the study in Egypt were indicated by Blake circle.

\section{Study Methodology}

The meat inspections techniques including ante-mortem inspections as well as post-mortem inspections were carried out by meat inspectors.

\section{ANTE-MORTEM INSPECTION}

Ante-mortem inspection was performed on the lairage. Where, animal behavior, illness marks and movements of animals were observed and noted as described by the Food and Agriculture Organization (FAO, 1994).

\section{POST-MORTEM INSPECTION}

The post-mortem inspection was carried out (FAO, 1994). Briefly, it was performed by visual examination, palpation and incisions of lymph nodes and organs including head, heart, lungs, kidneys, spleen, liver and intestine. Lesions were diagnosed based on pathological alterations including changes in the size, colour, consistency and existence of parasites or their lesions. An additional detailed inspection was performed in some illnesses like tuberculosis and cysticercosis. The judgments were categorized as fully approved and partially approved for human consumption. While 
the total condemnation decision was taken in case of generalized illness and/ or severe anomalies.

\section{ASSESSMENT OF FINANCIAL LOSS}

The direct economic loss in each abattoir was determined by the formula described by Khanjari et al. (2010).

$$
D E L=N \times P \times W
$$

DEL: Direct economic loss; N: number of condemned carcasses / and offals; P: Average price of carcasses /and offal ( $\mathrm{Kg} /$ Egyptian pound); W: average carcasses / and offal weight $(\mathrm{Kg})$.

The average price of the various carcasses and their offals was determined by their marketable cost in Alexandria, Qena and Aswancities during 2017-2018 (Table 1).

Table 1: Average prices of carcasses and their offals in Egypt during 2017-2018.

\begin{tabular}{|c|c|c|c|}
\hline Item & $\begin{array}{l}\text { Average } \\
\text { weight }(\mathrm{Kg})\end{array}$ & $\begin{array}{l}\text { Average price } \\
\text { (Egyptian } \\
\text { pound/ Kg) }\end{array}$ & $\begin{array}{l}\text { Total price } \\
\text { (Egyptian } \\
\text { pound) }\end{array}$ \\
\hline Cattle & 200 & 100 & 20000 \\
\hline Buffalo & 200 & 90 & 18000 \\
\hline Camel & 312.5 & 80 & 25000 \\
\hline Sheep & 40 & 125 & 5000 \\
\hline Bovine liver & 6.5 & 100 & 650 \\
\hline Liver camel & 8.5 & 100 & 850 \\
\hline Ovine liver & 1.2 & 100 & 120 \\
\hline Bovine lung & 4 & 75 & 300 \\
\hline Lung camel & 5 & 80 & 400 \\
\hline Ovine lung & 1.25 & 80 & 100 \\
\hline Bovine Kidneys & 1.6 & 75 & 120 \\
\hline Kidney camel & 3.5 & 80 & 280 \\
\hline Ovine Kidneys & 1 & 70 & 70 \\
\hline Bovine heart & 2.4 & 100 & 240 \\
\hline Heart camel & 3.8 & 100 & 380 \\
\hline Ovine heart & 0.5 & 100 & 50 \\
\hline Bovine spleen & 1.2 & 100 & 120 \\
\hline Spleen camel & 2.4 & 100 & 240 \\
\hline Ovine Spleen & 0.5 & 100 & 50 \\
\hline
\end{tabular}

1 USD was equal to 16 Egyptian pound.

\section{DATA ANALYSIS}

The data were analyzed by a Microsoft Excel spreadsheet. The $Z$ test was used to compare the proportions and when $p \leq 0.05$ referred to a significance.

\section{RESULTS AND DISCUSSION}

Abattoirs offered a significant chance to identify illness with financial values and zoonotic significance. Alexandria, Qena and Aswan abattoirs slaughtered 126686, 18886 and 22240 animals, respectively during the duration of this study. The variation in the numbers of slaughtered animals among the studied slaughterhouses may be attributed to the difference in the population size and their different economic status. Sheep followed by cattle, buffalo, goat and lastly camel meat was the preferred form of meat for Alexandrian individuals. Cattle meat is most preferable for individuals in Qena Governorate, followed by buffalo, sheep, camel and goat. Cattle is the first choice followed by camel, sheep and finally buffalo for people who lived in the Aswan.

\section{CAUSES OF CONDEMNATIONS OFWHOLE CARCASSES}

Out of 126686 animals were slaughtered in Alexandria abattoir $20(0.015 \%)$ animals were totally condemned due to various causes. Icterus was the predominate cause followed by fever, tuberculosis, ill bleeding and emaciation. Cattle, buffalo and sheep were the condemned animal species while there was no condemnation in camel and goat. All the total commended animals were native breeds, while there was not any total condemnation in the imported animals (Table 2). In Qena abattoir, out of 18886 livestock, only one sheep was completely condemned as a result of generalized tuberculosis and one cattle owing to ill bleeding (Table 3). Out of 22240 animals, 46 (0.20\%) were totally condemned in Aswan abattoir. Icterus, emaciation and generalized tuberculosis were the causes of condemnation at Aswan abattoir (Table 4).

In Aswan abattoir, the proportion of whole carcass rejection was significantly higher than in those in Alexandria and Qena abattoirs $(p<0.05)$.

In the current study, it is clear that there was no condemnation during the ante-mortem inspection, whereas all condemnations occurred as a result of diseases detected during post-mortem inspection. The obtained results were comparable with Tembo and Nonga (2015) who found that $0.05 \%$ of the animals slaughtered in Dodoma, Tanzania were completed commended as a result of tuberculosis, icterus and cysticercosis. The reasons for full carcass condemnation in the current work were almost identical to those recorded in Ethiopia (Mummed and Webb, 2015).

\section{PARTIAL CONDEMNATION OF THE CARCASS}

Localized affections of quarters of food animals with tuberculosis were the main cause of partial condemnation. Two cases of tuberculosis affection were detected at Alexandria abattoir (0.001\%) as illustrated in Table 2. While at Qena abattoir one cattle and two buffalo (0.015\%) (Table 3). Concerning Aswan abattoir, one case 
in cattle and 9 cases in camel carcass $(0.044 \%)$ (Table 4) were identified.

Table 2: Causes of condemnation of slaughtered animals in Alexandria slaughterhouse during 2017 and 2018.

\begin{tabular}{|c|c|c|c|c|c|c|c|c|c|c|c|c|c|}
\hline \multirow[t]{3}{*}{ Cause } & \multicolumn{6}{|l|}{2017} & \multicolumn{6}{|l|}{2018} & \multirow{3}{*}{$\begin{array}{l}\text { Total } \\
\mathrm{n}=126686\end{array}$} \\
\hline & \multicolumn{2}{|l|}{ Cattle } & \multirow{2}{*}{$\begin{array}{l}\text { Buffalo } \\
n=10809\end{array}$} & \multirow{2}{*}{$\begin{array}{l}\text { Camel } \\
n=16\end{array}$} & \multirow{2}{*}{$\begin{array}{l}\text { Sheep } \\
n=27363\end{array}$} & \multirow{2}{*}{$\begin{array}{l}\text { Goat } \\
n=46\end{array}$} & \multicolumn{2}{|l|}{ Cattle } & \multirow{2}{*}{$\begin{array}{l}\text { Buffalo } \\
\text { n=13936 }\end{array}$} & \multirow{2}{*}{$\begin{array}{l}\text { Camel } \\
n=4\end{array}$} & \multirow{2}{*}{$\begin{array}{l}\text { Sheep } \\
n=33393\end{array}$} & \multirow{2}{*}{$\begin{array}{l}\text { Goat } \\
n=86\end{array}$} & \\
\hline & $\begin{array}{l}\text { Nativen= } \\
15733\end{array}$ & $\begin{array}{l}\text { Import- } \\
\text { ed } n=340\end{array}$ & & & & & $\begin{array}{l}\text { Native } \\
n=24891\end{array}$ & $\begin{array}{l}\text { Import- } \\
\text { ed } n=69\end{array}$ & & & & & \\
\hline $\begin{array}{l}\text { Generalized } \\
\text { tuberculosis }\end{array}$ & $\begin{array}{l}1 \\
(0.006 \%)\end{array}$ & $\begin{array}{l}0 \\
(0 \%)\end{array}$ & 0 & 0 & 0 & 0 & $\begin{array}{l}1 \\
(0.004 \%)\end{array}$ & 0 & $\begin{array}{l}1 \\
(0.007 \%)\end{array}$ & 0 & 0 & 0 & $\begin{array}{l}3 \\
(0.002 \%)\end{array}$ \\
\hline $\begin{array}{l}\text { Tuberculosis } \\
\text { in quarter }\end{array}$ & $\begin{array}{l}1 \\
(0.006 \%)\end{array}$ & 0 & 0 & 0 & 0 & 0 & $\begin{array}{l}1 \\
(0.004 \%)\end{array}$ & 0 & 0 & 0 & 0 & 0 & $\begin{array}{l}2 \\
(0.001 \%)\end{array}$ \\
\hline Fever & $\begin{array}{l}0 \\
(0 \%)\end{array}$ & 0 & 0 & 0 & 0 & 0 & $\begin{array}{l}1 \\
(0.004 \%)\end{array}$ & 0 & $\begin{array}{l}1 \\
(0.007 \%)\end{array}$ & 0 & $\begin{array}{l}1 \\
(0.002 \%)\end{array}$ & 0 & $\begin{array}{l}3 \\
(0.002 \%)\end{array}$ \\
\hline Icterus & $\begin{array}{l}3 \\
(0.01 \%)\end{array}$ & 0 & $\begin{array}{l}1 \\
(0.009 \%)\end{array}$ & 0 & 0 & 0 & $\begin{array}{l}3 \\
(0.01 \%)\end{array}$ & 0 & $\begin{array}{l}1 \\
(0.007 \%)\end{array}$ & 0 & $\begin{array}{l}4 \\
(0.01 \%)\end{array}$ & 0 & $\begin{array}{l}12 \\
(0.009 \%)\end{array}$ \\
\hline Ill bleeding & 0 & 0 & 0 & 0 & 0 & 0 & 0 & 0 & $\begin{array}{l}1 \\
(0.007 \%)\end{array}$ & 0 & & 0 & $\begin{array}{l}1 \\
(0.0007 \%)\end{array}$ \\
\hline Emaciation & $\begin{array}{l}1 \\
(0.006 \%)\end{array}$ & 0 & & 0 & 0 & 0 & 0 & 0 & 0 & 0 & 0 & 0 & $\begin{array}{l}1 \\
(0.0007 \%)\end{array}$ \\
\hline \multicolumn{14}{|l|}{ Lung } \\
\hline Tuberculosis & $\begin{array}{l}67 \\
(0.42 \%)\end{array}$ & $\begin{array}{l}8 \\
(2.35 \%)\end{array}$ & $\begin{array}{l}77 \\
(0.71 \%)\end{array}$ & 0 & & 0 & $\begin{array}{l}104 \\
(0.41 \%)\end{array}$ & $\begin{array}{l}5 \\
(7.24 \%)\end{array}$ & $\begin{array}{l}109 \\
(0.78 \%)\end{array}$ & 0 & 0 & 0 & $\begin{array}{l}370 \\
(0.29 \%)\end{array}$ \\
\hline Congestion & $\begin{array}{l}39 \\
(0.24 \%)\end{array}$ & $\begin{array}{l}10 \\
(2.94 \%)\end{array}$ & $\begin{array}{l}38 \\
(0.35 \%)\end{array}$ & 0 & $\begin{array}{l}182 \\
(0.66 \%)\end{array}$ & 0 & $\begin{array}{l}80 \\
(0.32 \%)\end{array}$ & $\begin{array}{l}3 \\
(4.34 \%)\end{array}$ & $\begin{array}{l}54 \\
(0.38 \%)\end{array}$ & 0 & $\begin{array}{l}258 \\
(0.77 \%)\end{array}$ & 0 & $\begin{array}{l}664 \\
(0.52 \%)\end{array}$ \\
\hline $\begin{array}{l}\text { Parasitic } \\
\text { cyst }\end{array}$ & $\begin{array}{l}3(0.01 \%) \\
{ }^{*} \text {. bovis }\end{array}$ & $\begin{array}{l}3 \\
(0.88 \%) \\
\text { C. bovis }\end{array}$ & $\begin{array}{l}12 \\
(0.11 \%) \\
\text { Hydatid } \\
\text { cyst }\end{array}$ & 0 & $\begin{array}{l}155 \\
(0.56 \%) \\
\text { C. ovis }\end{array}$ & 0 & $\begin{array}{l}6(0.02 \%) \\
\text { C. bovis }\end{array}$ & 0 & $\begin{array}{l}10(0.07 \%) \\
\text { Hydatid } \\
\text { cyst }\end{array}$ & 0 & $\begin{array}{l}210 \\
(0.62 \%) \\
\text { C. ovis }\end{array}$ & 0 & $\begin{array}{l}399 \\
(0.31 \%)\end{array}$ \\
\hline Pneumonia & $\begin{array}{l}27 \\
(0.17 \%)\end{array}$ & $\begin{array}{l}5 \\
(1.47 \%)\end{array}$ & $\begin{array}{l}7 \\
(0.64 \%)\end{array}$ & 0 & $\begin{array}{l}141 \\
(0.51 \%)\end{array}$ & 0 & $\begin{array}{l}56 \\
(0.22 \%)\end{array}$ & & $\begin{array}{l}14 \\
(0.10 \%)\end{array}$ & 0 & $\begin{array}{l}253 \\
(0.75 \%)\end{array}$ & 0 & $\begin{array}{l}503 \\
(0.39 \%)\end{array}$ \\
\hline \multicolumn{14}{|l|}{ Heart } \\
\hline Cysticercus & $\begin{array}{l}65 \\
(0.41 \%) \\
\text { C. bovis }\end{array}$ & $\begin{array}{l}1 \\
(0.29 \%) \\
\text { C. bovis }\end{array}$ & $\begin{array}{l}4 \\
(0.03 \%) \\
\text { C. bovis }\end{array}$ & 0 & $\begin{array}{l}205 \\
(0.74 \%) \\
\text { C. ovis }\end{array}$ & 0 & $\begin{array}{l}4(0.01 \%) \\
\text { C. bovis }\end{array}$ & 0 & 0 & 0 & $\begin{array}{l}52(0.15 \%) \\
\text { C. ovis }\end{array}$ & 0 & $\begin{array}{l}331 \\
(0.26 \%)\end{array}$ \\
\hline $\begin{array}{l}\text { Traumatic } \\
\text { pericarditis }\end{array}$ & $\begin{array}{l}7 \\
(0.04 \%)\end{array}$ & & $\begin{array}{l}24 \\
(0.22 \%)\end{array}$ & 0 & $\begin{array}{l}44 \\
(0.16 \%)\end{array}$ & 0 & $\begin{array}{l}17 \\
(0.06 \%)\end{array}$ & 0 & $\begin{array}{l}32 \\
(0.22 \%)\end{array}$ & 0 & $\begin{array}{l}62 \\
(0.18 \%)\end{array}$ & 0 & $\begin{array}{l}186 \\
(0.14 \%)\end{array}$ \\
\hline \multicolumn{14}{|l|}{ Liver } \\
\hline Tuberculosis & $\begin{array}{l}15 \\
(0.09 \%)\end{array}$ & $\begin{array}{l}4 \\
(1.17 \%)\end{array}$ & $\begin{array}{l}28 \\
(0.25 \%)\end{array}$ & 0 & & 0 & $\begin{array}{l}32 \\
(0.12 \%)\end{array}$ & & $\begin{array}{l}44 \\
(0.31 \%)\end{array}$ & 0 & 0 & 0 & $\begin{array}{l}123 \\
(0.09 \%)\end{array}$ \\
\hline Cysticercus & $\begin{array}{l}3(0.19 \%) \\
\text { C. bovis }\end{array}$ & 0 & $\begin{array}{l}4 \\
(0.037 \%) \\
\text { C. bovis }\end{array}$ & 0 & $\begin{array}{l}33(0.12 \%) \\
\text { C. ovis }\end{array}$ & 0 & $\begin{array}{l}2 \\
(0.008 \%) \\
\text { C. bovis }\end{array}$ & $\begin{array}{l}2 \\
(2.89 \%) \\
\text { C. bovis }\end{array}$ & $\begin{array}{l}2(0.01 \%) \\
\text { C. bovis }\end{array}$ & 0 & $\begin{array}{l}30(0.08 \%) \\
\text { C. ovis }\end{array}$ & 0 & $\begin{array}{l}76 \\
(0.05 \%)\end{array}$ \\
\hline Abscesses & $\begin{array}{l}14 \\
(0.08 \%)\end{array}$ & $\begin{array}{l}5 \\
(1.47 \%)\end{array}$ & $\begin{array}{l}12 \\
(0.11 \%)\end{array}$ & 0 & $\begin{array}{l}57 \\
(0.20 \%)\end{array}$ & 0 & $\begin{array}{l}66 \\
(0.26 \%)\end{array}$ & $\begin{array}{l}4 \\
(5.79 \%)\end{array}$ & $\begin{array}{l}27 \\
(0.19 \%)\end{array}$ & & $\begin{array}{l}136 \\
(0.40 \%)\end{array}$ & 0 & $\begin{array}{l}321 \\
(0.25 \%)\end{array}$ \\
\hline Fasciola & $\begin{array}{l}49 \\
(0.31 \%)\end{array}$ & $\begin{array}{l}8 \\
(2.35 \%)\end{array}$ & $\begin{array}{l}35 \\
(0.32 \%)\end{array}$ & 0 & & 0 & $\begin{array}{l}68 \\
(0.27 \%)\end{array}$ & $\begin{array}{l}2 \\
(2.89 \%)\end{array}$ & $\begin{array}{l}53 \\
(0.38 \%)\end{array}$ & 0 & $\begin{array}{l}157 \\
(0.47 \%)\end{array}$ & 0 & $\begin{array}{l}372 \\
(0.09 \%)\end{array}$ \\
\hline \multicolumn{14}{|l|}{ Head } \\
\hline Cysticercus & $\begin{array}{l}23 \\
(0.14 \%) \\
\text { C. bovis }\end{array}$ & $\begin{array}{l}1 \\
(0.29 \%) \\
\text { C. bovis }\end{array}$ & $\begin{array}{l}4 \\
(0.03 \%) \\
\text { C. bovis }\end{array}$ & 0 & $\begin{array}{l}107 \\
(0.39 \%) \\
\text { C. bovis }\end{array}$ & 0 & $\begin{array}{l}4(0.01 \%) \\
\text { C. bovis }\end{array}$ & & & 0 & $\begin{array}{l}23(0.06 \%) \\
\text { C. ovis }\end{array}$ & 0 & $\begin{array}{l}162 \\
(0.12 \%)\end{array}$ \\
\hline Tuberculosis & $\begin{array}{l}14 \\
(0.08 \%)\end{array}$ & $\begin{array}{l}1 \\
(0.29 \%)\end{array}$ & $\begin{array}{l}22 \\
(0.20 \%)\end{array}$ & 0 & & 0 & $\begin{array}{l}38 \\
(0.15 \%)\end{array}$ & & $\begin{array}{l}16 \\
(0.11 \%)\end{array}$ & 0 & & 0 & $\begin{array}{l}91 \\
(0.07 \%)\end{array}$ \\
\hline Kidneys & & & & & & & & & & & & 0 & \\
\hline $\begin{array}{l}\text { Hydrone- } \\
\text { phrosis }\end{array}$ & $\begin{array}{l}4 \\
(0.02 \%)\end{array}$ & 0 & $\begin{array}{l}2 \\
(0.01 \%)\end{array}$ & 0 & & 0 & $\begin{array}{l}4 \\
(0.01 \%)\end{array}$ & & & 0 & & 0 & $\begin{array}{l}10 \\
(0.007 \%)\end{array}$ \\
\hline $\begin{array}{l}\text { Renal } \\
\text { fibrosis }\end{array}$ & $\begin{array}{l}14 \\
(0.08 \%)\end{array}$ & $\begin{array}{l}2 \\
(0.58 \%)\end{array}$ & $\begin{array}{l}32 \\
(0.29 \%)\end{array}$ & 0 & $\begin{array}{l}49 \\
(0.17 \%)\end{array}$ & 0 & $\begin{array}{l}67 \\
(0.26 \%)\end{array}$ & $\begin{array}{l}2 \\
(2.89 \%)\end{array}$ & $\begin{array}{l}44 \\
(0.31 \%)\end{array}$ & $\begin{array}{l}4 \\
(100 \%)\end{array}$ & $\begin{array}{l}64 \\
(0.19 \%)\end{array}$ & 0 & $\begin{array}{l}278 \\
(0.21 \%)\end{array}$ \\
\hline
\end{tabular}




\begin{tabular}{|c|c|c|c|c|c|c|c|c|c|c|c|c|c|}
\hline Abscesses & $3(0.01 \%)$ & 0 & 0 & 0 & 0 & 0 & 0 & 0 & 0 & 0 & & 0 & $3(0.002 \%)$ \\
\hline Spleen & & & & $\mathbf{0}$ & & & & & & & & & \\
\hline $\begin{array}{l}\text { Enlarge- } \\
\text { ment }\end{array}$ & $\begin{array}{l}64 \\
(0.40 \%)\end{array}$ & $\begin{array}{l}4 \\
(1.17 \%)\end{array}$ & $\begin{array}{l}35 \\
(0.32 \%)\end{array}$ & 0 & $\begin{array}{l}117 \\
(0.42 \%)\end{array}$ & 0 & $\begin{array}{l}158 \\
(0.63 \%)\end{array}$ & $\begin{array}{l}3 \\
(4.34 \%)\end{array}$ & $\begin{array}{l}77 \\
(0.55 \%)\end{array}$ & 0 & $\begin{array}{l}155 \\
(0.46 \%)\end{array}$ & 0 & $\begin{array}{l}613 \\
(0.48 \%)\end{array}$ \\
\hline Congestion & $\begin{array}{l}17 \\
(0.10 \%)\end{array}$ & $\begin{array}{l}1 \\
(0.29 \%)\end{array}$ & $\begin{array}{l}12 \\
(0.11 \%)\end{array}$ & 0 & $\begin{array}{l}59 \\
(0.21 \%)\end{array}$ & 0 & $\begin{array}{l}8 \\
(0.03 \%)\end{array}$ & 0 & $\begin{array}{l}4 \\
(0.02 \%)\end{array}$ & 0 & $51(0.15 \%)$ & 0 & $\begin{array}{l}152 \\
(0.11 \%)\end{array}$ \\
\hline \multicolumn{14}{|l|}{ Intestine } \\
\hline Tuberculosis & $\begin{array}{l}7 \\
(0.04 \%)\end{array}$ & 0 & $\begin{array}{l}280 \\
(2.59 \%)\end{array}$ & 0 & & 0 & $\begin{array}{l}35 \\
(0.14 \%)\end{array}$ & 0 & $\begin{array}{l}242 \\
(1.73 \%)\end{array}$ & 0 & 0 & 0 & $\begin{array}{l}564 \\
(0.44 \%)\end{array}$ \\
\hline Congestion & $\begin{array}{l}20 \\
(0.12 \%)\end{array}$ & 0 & $\begin{array}{l}24 \\
(0.22 \%)\end{array}$ & 0 & $\begin{array}{l}101 \\
(0.36 \%)\end{array}$ & 0 & $\begin{array}{l}52 \\
(0.20 \%)\end{array}$ & 0 & $\begin{array}{l}32 \\
(0.22 \%)\end{array}$ & 0 & $\begin{array}{l}122 \\
(0.36 \%)\end{array}$ & 0 & $\begin{array}{l}351 \\
(0.27 \%)\end{array}$ \\
\hline $\begin{array}{l}\text { Emergency } \\
\text { slaughter }\end{array}$ & $\begin{array}{l}1 \\
(0.006 \%)\end{array}$ & 0 & $\begin{array}{l}1 \\
(0.009 \%)\end{array}$ & 0 & & 0 & $\begin{array}{l}11 \\
(0.044 \%)\end{array}$ & 0 & $\begin{array}{l}4 \\
(0.028 \%)\end{array}$ & 0 & 0 & 0 & $\begin{array}{l}17 \\
(0.01 \%)\end{array}$ \\
\hline
\end{tabular}

${ }^{*} C$ : Cysticercus.

Table 3: Causes of condemnation of slaughtered animals in Qena slaughterhouse during 2017 and 2018.

\begin{tabular}{|c|c|c|c|c|c|c|c|c|c|c|c|}
\hline \multirow[t]{3}{*}{ Cause } & \multicolumn{5}{|l|}{2017} & \multicolumn{5}{|l|}{2018} & \multirow{3}{*}{$\begin{array}{l}\text { Total } \\
n=18886\end{array}$} \\
\hline & Cattle & Buffalo & Camel & Sheep & Goat & Cattle & Buffalo & Camel & Sheep & Goat & \\
\hline & $\mathrm{n}=8256$ & $\mathrm{n}=653$ & $n=9$ & $\mathrm{n}=464$ & $\mathrm{n}=7$ & $\mathrm{n}=8419$ & $\mathrm{n}=569$ & $\mathrm{n}=24$ & $\mathrm{n}=482$ & $\mathrm{n}=3$ & \\
\hline $\begin{array}{l}\text { Generalized } \\
\text { tuberculosis }\end{array}$ & 0 & 0 & 0 & $1(0.21 \%)$ & 0 & 0 & 0 & 0 & 0 & 0 & $1(0.005 \%)$ \\
\hline $\begin{array}{l}\text { Tuberculosis in } \\
\text { quarter }\end{array}$ & 0 & $\begin{array}{l}1 \\
(0.15 \%)\end{array}$ & 0 & 0 & 0 & $\begin{array}{l}1 \\
(0.01 \%)\end{array}$ & $\begin{array}{l}1 \\
(0.17 \%)\end{array}$ & 0 & 0 & 0 & $\begin{array}{l}3 \\
(0.01 \%)\end{array}$ \\
\hline Ill bleeding & 0 & 0 & 0 & 0 & 0 & $1(0.01 \%)$ & 0 & 0 & 0 & 0 & $1(0.005 \%)$ \\
\hline Emaciation & 0 & 0 & 0 & 0 & 0 & 0 & 0 & 0 & 0 & 0 & \\
\hline $\begin{array}{l}\text { Abscesses in } \\
\text { muscle }\end{array}$ & $2(0.02 \%)$ & 0 & 0 & $1(0.21 \%)$ & 0 & $3(0.03 \%)$ & 0 & 0 & 0 & 0 & $6(0.03 \%)$ \\
\hline \multicolumn{12}{|l|}{ Lung } \\
\hline Tuberculosis & 0 & 0 & 0 & 0 & 0 & 0 & 0 & 0 & 0 & 0 & \\
\hline Congestion & 0 & 0 & 0 & $1(0.21 \%)$ & 0 & 0 & 0 & 0 & 0 & 0 & $1(0.005 \%)$ \\
\hline Pneumonia & 0 & 0 & 1(11.11\%) & $3(0.64 \%)$ & 0 & $3(0.03 \%)$ & 0 & 0 & $2(0.41 \%)$ & 0 & $9(0.04 \%)$ \\
\hline Abscesses & 0 & 0 & 0 & 0 & 0 & $1(0.01 \%)$ & 0 & $1(4.16 \%)$ & $1(0.20 \%)$ & 0 & $3(0.01 \%)$ \\
\hline Heart & & 0 & & & & & & & & & \\
\hline Cysticercus & 0 & 0 & 0 & $\begin{array}{l}3(0.64 \%) \\
\text { C. ovis }\end{array}$ & 0 & 0 & 0 & 0 & 0 & 0 & $\begin{array}{l}3 \\
(0.01 \%)\end{array}$ \\
\hline $\begin{array}{l}\text { Traumatic } \\
\text { pericarditis }\end{array}$ & 0 & 0 & 0 & 0 & 0 & 0 & $1(0.17 \%)$ & 0 & 0 & 0 & $1(0.005 \%)$ \\
\hline Congestion & 0 & 0 & 0 & 0 & 0 & $3(0.03 \%)$ & 0 & 0 & 0 & 0 & $3(0.01 \%)$ \\
\hline \multicolumn{12}{|l|}{ Liver } \\
\hline Fasciola & $47(0.56 \%)$ & $6(0.91 \%)$ & 0 & $2(0.43 \%)$ & 0 & $41(0.48 \%)$ & $6(1.05 \%)$ & & $1(0.20 \%)$ & & $103(0.54 \%)$ \\
\hline \multicolumn{12}{|l|}{ Head } \\
\hline Cysticercus & $\begin{array}{l}1(0.01 \%) \\
\text { C. bovis }\end{array}$ & 0 & 0 & 0 & 0 & 0 & 0 & 0 & 0 & 0 & $1(0.005 \%)$ \\
\hline Tuberculosis & $13(0.15 \%)$ & 0 & 0 & 0 & 0 & $10(0.11 \%)$ & 0 & 0 & 0 & 0 & $23(0.12 \%)$ \\
\hline Kidney & 0 & 0 & 0 & 0 & 0 & 0 & 0 & 0 & 0 & 0 & 0 \\
\hline Spleen & 0 & 0 & 0 & 0 & 0 & 0 & 0 & 0 & 0 & 0 & 0 \\
\hline Intestine & 0 & 0 & 0 & 0 & 0 & 0 & 0 & 0 & 0 & 0 & 0 \\
\hline
\end{tabular}


Fracture and 0

$0 \quad 0$

$0 \quad 0$

$1(0.011 \%) 0$

0

$0 \quad 1(0.005 \%)$

contusion

Table 4: Causes of condemnation of slaughtered animals in Aswan slaughterhouse during 2017 and 2018.

\begin{tabular}{|c|c|c|c|c|c|c|c|c|c|}
\hline \multirow[t]{3}{*}{ Cause } & \multicolumn{4}{|l|}{2017} & \multicolumn{4}{|l|}{2018} & \multirow{3}{*}{$\begin{array}{l}\text { Total } \\
n=22240\end{array}$} \\
\hline & Cattle & Buffalo & Camel & Sheep & Cattle & Buffalo & Camel & Sheep & \\
\hline & $\mathrm{n}=5720$ & $\mathrm{n}=88$ & $\mathrm{n}=2054$ & $\mathrm{n}=835$ & $\mathrm{n}=11214$ & $n=202$ & $\mathrm{n}=574$ & $n=1553$ & \\
\hline $\begin{array}{l}\text { Generalized } \\
\text { tuberculosis }\end{array}$ & 0 & 0 & 0 & $1(0.11 \%)$ & 0 & 0 & 0 & 0 & $1(0.004 \%)$ \\
\hline $\begin{array}{l}\text { Tuberculosis } \\
\text { in quarter }\end{array}$ & $1(0.01 \%)$ & 0 & $5(0.24 \%)$ & 0 & 0 & 0 & $4(0.69 \%)$ & 0 & $10(0.044 \%)$ \\
\hline Icterus & $2(0.34 \%)$ & $1(1.13 \%)$ & 0 & $7(0.835 \%)$ & $20(0.17 \%)$ & 00 & 0 & $12(0.77 \%)$ & $42(0.18 \%)$ \\
\hline Emaciation & 0 & 0 & 0 & $3(0.35 \%)$ & 0 & 0 & 0 & 0 & $3(0.013 \%)$ \\
\hline \multicolumn{10}{|l|}{ Lung } \\
\hline Tuberculosis & $\begin{array}{l}18 \\
(0.31 \%)\end{array}$ & $\begin{array}{l}5 \\
(5.68 \%)\end{array}$ & $10(0.48 \%)$ & 0 & $\begin{array}{l}29 \\
(0.25 \%)\end{array}$ & 0 & $\begin{array}{l}12 \\
(2.09 \%)\end{array}$ & 0 & $\begin{array}{l}74 \\
(0.33 \%)\end{array}$ \\
\hline Congestion & $\begin{array}{l}65 \\
(1.13 \%)\end{array}$ & $\begin{array}{l}15 \\
(17.04 \%)\end{array}$ & $\begin{array}{l}141 \\
(6.86 \%)\end{array}$ & 0 & $\begin{array}{l}198 \\
(1.76 \%)\end{array}$ & $\begin{array}{l}43 \\
(21.28 \%)\end{array}$ & 193(33.62\%) & 0 & $655(2.94 \%)$ \\
\hline Parasitic cysts & 0 & 0 & $\begin{array}{l}256(12.46 \%) \\
\text { Hydatid cyst }\end{array}$ & 0 & $\begin{array}{l}65(0.57 \%) \\
\text { C. bovis }\end{array}$ & 0 & $\begin{array}{l}176(30.66 \%) \\
\text { Hydatid cyst }\end{array}$ & 0 & $497(2.23 \%)$ \\
\hline Pneumonia & 0 & 0 & $16(0.77 \%)$ & $7(0.83 \%)$ & 0 & 0 & 0 & 0 & $23(0.10 \%)$ \\
\hline \multicolumn{10}{|l|}{ Heart } \\
\hline Cysticercus & $\begin{array}{l}376(6.57 \%) \\
\text { C. bovis }\end{array}$ & 0 & 0 & $\begin{array}{l}\text { 55(6.58\%) } \\
\text { C. ovis }\end{array}$ & $\begin{array}{l}361(3.21 \%) \\
\text { C. bovis }\end{array}$ & 0 & $\begin{array}{l}1(0.17 \%) \\
\text { C. cameli }\end{array}$ & $\begin{array}{l}130(8.37 \%) \\
\text { C. ovis }\end{array}$ & $923(4.15 \%)$ \\
\hline \multicolumn{10}{|l|}{ Liver } \\
\hline Cysticercus & 0 & 0 & $\begin{array}{l}16(0.77 \%) \\
\text { C. cameli }\end{array}$ & $\begin{array}{l}7(0.83 \%) \\
\text { C.ovis }\end{array}$ & $23(0.20 \%)$ & 7 & $\begin{array}{l}13(2.26 \%) \\
\text { C. cameli }\end{array}$ & $3(0.19 \%)$ & $69(0.310 \%)$ \\
\hline Fasciola & $\begin{array}{l}1302 \\
(22.76 \%)\end{array}$ & $\begin{array}{l}36 \\
(40.9 \%)\end{array}$ & 0 & $\begin{array}{l}168 \\
(20.11 \%)\end{array}$ & $\begin{array}{l}1458 \\
(13 \%)\end{array}$ & $\begin{array}{l}54 \\
(26.73 \%)\end{array}$ & 0 & $\begin{array}{l}208 \\
(13.39 \%)\end{array}$ & $\begin{array}{l}3226 \\
(14.50 \%)\end{array}$ \\
\hline \multicolumn{10}{|l|}{ Head } \\
\hline Cysticercus & $\begin{array}{l}150(2.62 \%) \\
\text { C. bovis }\end{array}$ & 0 & 0 & 0 & $\begin{array}{l}78(0.69 \%) \\
\text { C. bovis }\end{array}$ & 0 & 0 & 0 & $228(1.02 \%)$ \\
\hline Tuberculosis & $13(0.22 \%)$ & 0 & 0 & 0 & $17(0.15 \%)$ & 0 & 0 & 0 & $30(0.134 \%)$ \\
\hline \multicolumn{10}{|l|}{ Kidney } \\
\hline $\begin{array}{l}\text { Hydrone- } \\
\text { phrosis }\end{array}$ & $186(3.25 \%)$ & $4(4.54 \%)$ & $72(3.50 \%)$ & $20(2.39 \%)$ & $217(1.93 \%)$ & $10(1.93 \%)$ & $19(3.31 \%)$ & 0 & $528(2.37 \%)$ \\
\hline $\begin{array}{l}\text { Intestinal } \\
\text { Congestion }\end{array}$ & 0 & 0 & 0 & 0 & $12(0.10 \%)$ & $3(1.48 \%)$ & $7(1.21 \%)$ & 0 & $22(0.09 \%)$ \\
\hline
\end{tabular}

Table 5: Economic loss due to meat condemnation in Alexandria, Qena and Aswan slaughterhouses during 2017-2018.

$\begin{array}{llllllll}\begin{array}{l}\text { Con- } \\ \text { demned } \\ \text { part }\end{array} & \begin{array}{l}\text { Cause for con- } \\ \text { demnation }\end{array} & \begin{array}{l}\text { Alexandria } \\ \text { Number of } \\ \text { animals }\end{array} & \begin{array}{l}\text { Economic } \\ \text { loss (Egyp- } \\ \text { tian pound) }\end{array} & \begin{array}{l}\text { Number } \\ \text { of animals }\end{array} & \begin{array}{l}\text { Economic loss } \\ \text { (Egyptian pound) }\end{array} & \begin{array}{l}\text { Number of } \\ \text { animals }\end{array} & \begin{array}{l}\text { Economic } \\ \text { loss (Egyp- } \\ \text { tian pound) }\end{array}\end{array}$




\begin{tabular}{|c|c|c|c|c|c|c|c|c|}
\hline \multirow[t]{5}{*}{$\begin{array}{l}\text { Whole } \\
\text { carcass }\end{array}$} & $\begin{array}{l}\text { Generalized } \\
\text { tuberculosis }\end{array}$ & $\begin{array}{l}2 \text { cattle } \\
1 \text { buffalo }\end{array}$ & $\begin{array}{l}40000 \\
18000\end{array}$ & 1 sheep & 5000 & 1 sheep & 5000 & 68000 \\
\hline & Fever & $\begin{array}{l}1 \text { cattle } \\
1 \text { buffalo } \\
1 \text { sheep }\end{array}$ & $\begin{array}{l}20000 \\
18000 \\
5000\end{array}$ & 0 & 0 & 0 & 0 & 43000 \\
\hline & Icterus & $\begin{array}{l}6 \text { cattle } \\
2 \text { buffalo } \\
4 \text { sheep }\end{array}$ & $\begin{array}{l}120000 \\
3000 \\
20000\end{array}$ & 0 & 0 & $\begin{array}{l}22 \text { cattle } \\
1 \text { buffalo } \\
19 \text { sheep }\end{array}$ & $\begin{array}{l}440000 \\
18000 \\
95000\end{array}$ & 696000 \\
\hline & Ill bleeding & 1 buffalo & 18000 & 1 cattle & 18000 & 0 & 0 & 36000 \\
\hline & emaciation & 1 cattle & 20000 & 0 & 0 & 3 sheep & 15000 & 35000 \\
\hline $\begin{array}{l}\text { T.B in } \\
\text { Quarter }\end{array}$ & & 2 cattle & 10000 & $\begin{array}{l}1 \text { cattle } \\
2 \text { buffalo }\end{array}$ & $\begin{array}{l}5000 \\
9000\end{array}$ & $\begin{array}{l}1 \text { cattle } \\
9 \text { camel }\end{array}$ & $\begin{array}{l}5000 \\
56250\end{array}$ & 85250 \\
\hline \multirow[t]{5}{*}{ lung } & Tuberculosis & $\begin{array}{l}184 \text { lungs cattle } \\
186 \text { lungs buffalo }\end{array}$ & $\begin{array}{l}55200 \\
55800\end{array}$ & 0 & 0 & $\begin{array}{l}47 \text { lungs cattle } \\
5 \text { lungs buffalo } \\
22 \text { lungs camel }\end{array}$ & $\begin{array}{l}14100 \\
1500 \\
8800\end{array}$ & 135400 \\
\hline & congestion & $\begin{array}{l}132 \text { lungs cattle } \\
92 \text { lungs buffalo } \\
440 \text { lungs sheep }\end{array}$ & $\begin{array}{l}39600 \\
27600 \\
44000\end{array}$ & 1 lung sheep & 100 & $\begin{array}{l}263 \text { lungs cattle } \\
58 \text { lungs buffalo } \\
334 \text { lungs camel }\end{array}$ & $\begin{array}{l}78900 \\
17400 \\
133600\end{array}$ & 341200 \\
\hline & Parasitic cyst & $\begin{array}{l}12 \text { lungs cattle } \\
22 \text { lungs buffalo } \\
210 \text { lungs sheep }\end{array}$ & $\begin{array}{l}3600 \\
6600 \\
21000\end{array}$ & 0 & 0 & $\begin{array}{l}65 \text { lungs cattle } \\
432 \text { lungs camel }\end{array}$ & $\begin{array}{l}19500 \\
172800\end{array}$ & 223500 \\
\hline & Pneumonia & $\begin{array}{l}88 \text { lungs cattle } \\
21 \text { lungs buffalo } \\
394 \text { lungs sheep }\end{array}$ & $\begin{array}{l}26400 \\
6300 \\
39400\end{array}$ & $\begin{array}{l}3 \text { lungs } \\
\text { cattle } \\
1 \text { lungs } \\
\text { camel } \\
\text { 5lungs } \\
\text { sheep }\end{array}$ & $\begin{array}{l}900 \\
400 \\
500\end{array}$ & $\begin{array}{l}16 \text { lungs camel } \\
7 \text { lungs sheep }\end{array}$ & $\begin{array}{l}6400 \\
700\end{array}$ & 81000 \\
\hline & Abscesses & 0 & 0 & $\begin{array}{l}1 \text { lung cattle } \\
1 \text { lung } \\
\text { camel } \\
1 \text { lung sheep }\end{array}$ & $\begin{array}{l}300 \\
400 \\
100\end{array}$ & 0 & 0 & 800 \\
\hline \multirow[t]{3}{*}{ Heart } & Cysticercus & $\begin{array}{l}70 \text { hearts cattle } \\
4 \text { hearts buffalo } \\
257 \text { hearts sheep }\end{array}$ & $\begin{array}{l}16800 \\
960 \\
12850\end{array}$ & $\begin{array}{l}3 \text { hearts } \\
\text { sheep }\end{array}$ & 150 & $\begin{array}{l}737 \text { hearts cattle } \\
1 \text { hearts camel } \\
185 \text { hearts sheep }\end{array}$ & $\begin{array}{l}176880 \\
380 \\
9250\end{array}$ & 217270 \\
\hline & $\begin{array}{l}\text { Traumatic peri- } \\
\text { carditis }\end{array}$ & $\begin{array}{l}24 \text { hearts cattle } \\
56 \text { hearts buffalo } \\
106 \text { hearts sheep }\end{array}$ & $\begin{array}{l}5760 \\
13440 \\
5300\end{array}$ & $\begin{array}{l}1 \text { hearts } \\
\text { buffalo }\end{array}$ & 240 & 0 & 0 & 24740 \\
\hline & Congestion & 0 & 0 & $\begin{array}{l}3 \text { hearts } \\
\text { cattle }\end{array}$ & 720 & 0 & 0 & 720 \\
\hline \multirow[t]{4}{*}{ Liver } & Tuberculosis & $\begin{array}{l}51 \text { livers cattle } \\
72 \text { livers buffalos }\end{array}$ & $\begin{array}{l}33150 \\
46800\end{array}$ & 0 & 0 & 0 & 0 & 79950 \\
\hline & Cysticercus & $\begin{array}{l}7 \text { livers cattle } \\
6 \text { livers buffalos } \\
63 \text { livers sheep }\end{array}$ & $\begin{array}{l}4550 \\
3900 \\
7560\end{array}$ & 0 & 0 & $\begin{array}{l}23 \text { livers cattle } \\
7 \text { livers buffalo } \\
29 \text { livers camel } \\
10 \text { livers sheep }\end{array}$ & $\begin{array}{l}14950 \\
4550 \\
24650 \\
1200\end{array}$ & 61360 \\
\hline & Abscesses & $\begin{array}{l}89 \text { livers cattle } \\
39 \text { livers buffalo } \\
193 \text { livers sheep }\end{array}$ & $\begin{array}{l}57850 \\
25350 \\
23160\end{array}$ & 0 & 0 & 0 & 0 & 106360 \\
\hline & Fasciola & $\begin{array}{l}127 \text { livers cattle } \\
88 \text { livers buffalo } \\
157 \text { livers sheep }\end{array}$ & $\begin{array}{l}82550 \\
57200 \\
18840\end{array}$ & $\begin{array}{l}88 \text { livers } \\
\text { cattle } \\
12 \text { livers } \\
\text { buffalo } \\
3 \text { livers } \\
\text { sheep }\end{array}$ & $\begin{array}{l}57200 \\
7800 \\
360\end{array}$ & $\begin{array}{l}2760 \text { livers cattle } \\
90 \text { liver buffalo } \\
376 \text { liver sheep }\end{array}$ & $\begin{array}{l}1794000 \\
58500 \\
45120\end{array}$ & 2121570 \\
\hline kidneys & Hydronephrosis & $\begin{array}{l}8 \text { kidneys cattle } \\
2 \text { kidneys buffalos }\end{array}$ & $\begin{array}{l}960 \\
240\end{array}$ & 0 & 0 & $\begin{array}{l}403 \text { kidneys cattle } \\
14 \text { kidneys buffalo } \\
91 \text { kidneys camel } \\
20 \text { kidneys sheep }\end{array}$ & $\begin{array}{l}48360 \\
1680 \\
25480 \\
1400\end{array}$ & 78120 \\
\hline
\end{tabular}




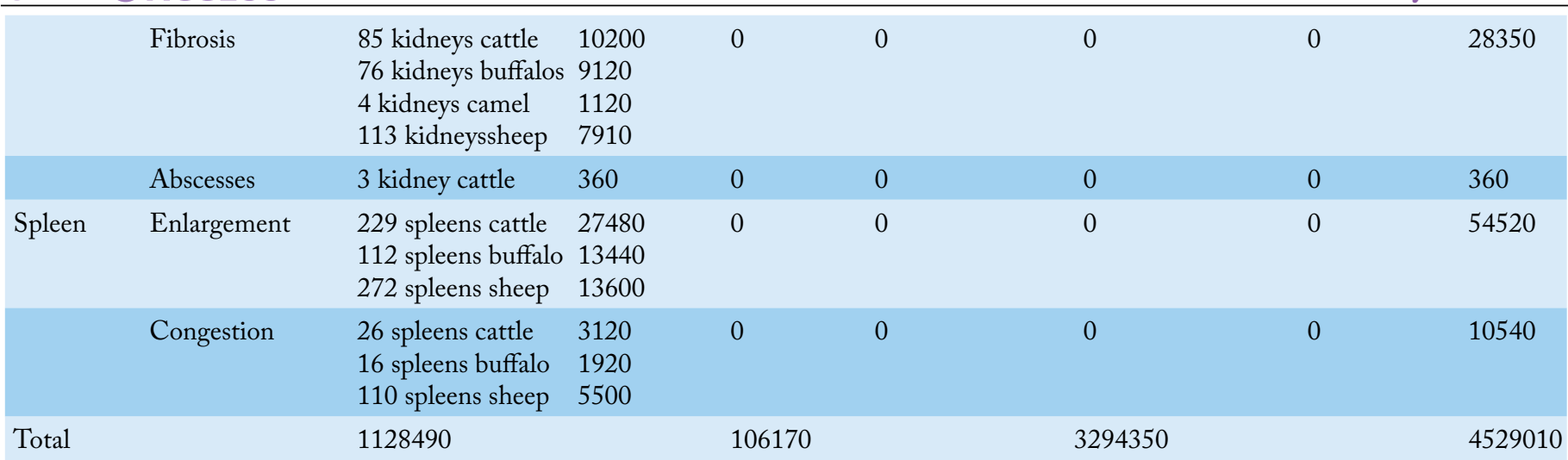

The proportion of partial carcass condemnation in Aswan abattoir was significantly higher than Alexandria and Qena abattoirs $(p<0.05)$. Tuberculosis is a serious disease of Egyptian livestock and has been shown to occur in various cities. Many reports demonstrated the presence of tuberculosis in the meat, Yibar et al. (2015) showed that the prevalence of tuberculosis in the examined cattle carcasses was $1.32 \%$ in Turkey. Therefore, the strict control program is required to reduce the prevalence of tuberculosis.

\section{CAUSES OF CONDEMNATIONS OF LUNGS}

In the current work, the most pathological affections were identified in the lungs (3198 lesions), which may be attributed to their structure and their direct connection with the external environment.

The total lung lesions in Alexandria, Qena and Aswan abattoirs were 1936 (1.52\%), 13 (0.068\%), 1249 (5.61\%), respectively. Based on statistical analysis, a significant difference was detected between the proportions of lung lesions among three abattoirs.

Congestion $(0.52 \%)$, pneumonia $(0.39 \%)$, parasitic cysts $(0.31 \%)$, and tuberculosis $(0.29 \%)$ were the primary cause of lung condemnation at Alexandria abattoir (Table 2). Moreover, pneumonia $(0.047 \%)$ and the formation of abscesses $(0.015 \%)$ were identified at Qena abattoir (Table 3). In Aswan abattoir, Congestion (2.94\%), parasitic cysts (2.23\%) and pneumonia (0.103\%) were recorded (Table 4). The parasitic cysts in Alexandria abattoir were categorized as cysticercosis in cattle and sheep while hydatid cysts were detected in buffalo. With regard to Aswan abattoir, hydatid cysts were observed in camel lungs and Cysticercus bovis was detected in cattle lung. The occurrence of hydatid cyst in the current work with high frequency in Aswan abattoir had a public health concern with financial importance particularly, in the rural areas where comprehensive grazing is carried out.

The obtained findings agreed with Ahmed et al. (2013) who reported that pneumonia was the major cause for lung condemnation in the inspected livestock in Ismailia abattoir, Egypt. The proportion of lungs condemnations in Dodoma, Tanzania abattoir was $10.5 \%$, emphysema, hydatid cyst and hemorrhage were the chief reasons for the condemnations (Tembo and Nonga, 2015). Moreover, an abattoir survey was performed in Iran, cattle were the most infected animals with hydatid cyst, followed by sheep and goats, while the most infected offals were livers followed by lungs (Ghasemian et al., 2018). Pezeshki et al. (2018) recorded that the prevalence of hydatidosis in cattle and sheep in 2015-2018 was $2.25 \%$ and $2.48 \%$, respectively in Tehran, Iran. The diversity of hydatidosis incidence in various countries may be attributed to county location, presence of dogs and degree of animal hygiene (Azami et al., 2013). Maxwell (2005) examined 5369 cattle in Nigeria and reported that $8.5 \%$ of the total lungs were condemned. Tuberculosis, pneumonia, abscesses and parasitic infection were the main causes of condemnation. Current work has shown that lungs lesions can cause numerous diseases for humans and have a serious impact on animal production. Some stressors factors as polluted air, fatigue, overpopulation in the farms were responsible for respiratory illness.

\section{CAUSES OF CONDEMNATIONS OF HEARTS}

The overall heart lesions in Alexandria, Qena and Aswan abattoirs were 517 (0.40\%), 7 (0.037\%) and 923 (4.15\%), respectively. Based on statistical analysis, significance difference was detected between the proportions of heart lesions among three abattoirs.

Traumatic pericarditis and parasitic infection were the main causes of heart condemnation at Alexandria and Qena abattoir. While the parasitic infection was the only cause for condemnation of the heart at the Aswan abattoir. All parasitic cysts have been recognized in all abattoirs as cysticercosis.

Traumatic pericarditis is comparatively widespread and caused by the swallowing of foreign bodies. Ahmed et al. (2013) noted that $8 \%$ of examined heart in Ismailia abattoir, Egypt were condemned as a result of heart 
adhesions and traumatic pericarditis. Comparably Jibat et al. (2008) have reported that out of 2688 animals were slaughtered at Debre Zeit, Ethiopia, 214 (7.9\%) hearts were condemned as a result of pericarditis, calcification and parasitic infection.

\section{CAUSES OF CONDEMNATIONS OF LIVERS}

The total liver lesions in Alexandria, Qena and Aswan were 892 (0.70\%), 103 (0.54\%) and 3295 (14.81\%), respectively. Based on statistical analysis, a significant difference was observed between the proportions of livers lesions among three abattoirs.

The chief causes for condemnations of livers at Alexandria abattoir were fasciola (0.29\%), abscesses (0.25\%), tuberculosis (0.09\%) and cysticercosis (0.05\%). While at Aswan abattoir, fasciola (14.50\%) and cysticercosis $(0.31 \%)$ were the primary causes of liver condemnation. In addition, fasciola infection (0.54\%) was the only cause for livers condemnations at Qena abattoir. Moreover, all parasitic cysts in the current study have been identified as cysticercosis in Alexandria and Aswan abattoirs.

The obtained data showed that fascioliasis is widespread in Egyptian slaughterhouses. Aminzare et al. (2018) obtained similar findings in the examined animals in Khorasan Razavi, Iran. Pezeshki et al. (2018) recorded that the prevalence of fascioliasis in cattle and sheep was $0.62 \%$ and $0.25 \%$, respectively in a study conducted in Tehran, Iran. The incidence of fasciolosis in sheep and goats was reported in different counties, Pakistan (51.3\% and 14.8\%) and Saudi Arabia (0.04\% and 0.00\%). In Tanzania, there was a high incidence of fasciola (30\%) (Nzalawahe and Komba, 2013). The high incidence of fascioliasis in the current study may be attributable to the appropriateness of climate conditions and the presence of snails. Infection with fasciola is widespread and characteristic in many African countries. Nevertheless, fascioliasis infrequently leads to death in farm animals and its impacts had led to decrease the outputs of animals and the rejection of livers in slaughterhouses (Kambarage et al., 1995).

Among 9880 cattle slaughtered in the Ismailia abattoir, 181cattle had livers abscess and 79 cattle had hepatic cirrhosis (Ahmed et al., 2013). Moreover, fascioliasis, cirrhosis and abscesses were the reasons for liver rejection in Zaria slaughterhouse (Raji et al., 2010). A study was conducted in three slaughterhouses in South Africa showed that the major causes of liver condemnation were fascioliasis, fibrosis and abscesses formation with associated financial loss USD 4527107 (Jaja et al., 2017). Liver lesions of food animals have an adverse effect on the economy via condemnation of diseased livers at the slaughterhouse in addition to their impact on the animal gain.
CAUSES OF CONDEMNATIONS OF HEADS

The overall head lesions at Alexandria, Qena and Aswan abattoirs were 253 (0.19\%), 24 (0.12\%) and 258 (1.16\%). Based on statistical analysis, a significant difference was identified between the proportions of heads lesions among three abattoirs.

Cysticercosis and tuberculosis were the main causes of condemnation of heads at abattoirs in Alexandria, Qena and Aswan in the present research. The bovine heads were the predominate species that were condemned among other slaughtered species.

The causes of condemnation of head in Ethiopia were contusion, parasitic infection and abscess formation (Mummed and Webb, 2015). Moreover, Beyene and Kiko (2019) stated that the prevalence of Cysticercus bovis was high in shoulder muscle followed by head muscle and cardiac muscle in slaughtered cattle in Ethiopia. The prevalence of condemnations of heads of cattle in Dodoma, Tanzania was $0.004 \%$ as a result of abscesses formation (Tembo and Nonga, 2015). It is important to state that the heads of food animals are not routinely split in Egyptian slaughterhouses and splitting is performed in suspected cases.

\section{CAUSES OF CONDEMNATIONS OF KIDNEYS}

At Aswan abattoir, the overall kidneys lesions were 528 (2.37\%) that were significantly greater than those at Alexandria abattoir 291 (0.22\%). The inspected kidneys were free from all kinds of affections at Qena abattoir.

The majorcausesfor condemnations of kidneysatAlexandria abattoir were fibrosis $(0.21 \%)$ and hydronephrosis $(0.008 \%)$ (Table 2). While the hydronephrosis was the only cause for kidneys condemnation at Aswan abattoir (Table 4).

Tavassoly (2003) achieved comparable findings. While, nephritis was the major cause for condemnation of $6.8 \%$ of inspected kidneys in Ethiopia (Jibat et al., 2008). Ahmed et al. (2013) reported that the kidney affections included crystals, white spots, pyelonephritis, fibrosis and hydronephrosis were identified among 9880 cattle slaughtered at Ismailia abattoir, Egypt, with an incidence of $1.01 \%, 0.98 \%, 0.52 \%, 0.38 \%$ and $0.23 \%$, respectively. In Dodoma, Tanzania, the incidence of condemned kidneys was $3.8 \%$ and the chief causes for condemnations were congenial renal cysts with hydronephrosis (Tembo and Nonga, 2015). 


\section{CAUSES OF CONDEMNATIONS OF SPLEENS}

In Alexandria abattoir, 765 (0.60\%) splenic lesions were observed. The lesions were classified into splenomegaly $(0.48 \%)$ and congestion $(0.001 \%)$ of spleens (Table 2$)$. However, Spleens were free from any affection in Qena and Aswan abattoirs. The primary cause of splenomegaly may be due to infection with blood parasites (Gracey et al., 1999). Meanwhile, 120 cattle out of 9880 showed splenomegaly at Ismailia abattoir, Egypt (Ahmed et al., 2013). Splenomegaly, splenitis and contamination were the key reasons for the condemnation of spleen in Tanzania (Mellau et al., 2011). Nevertheless, in some cases in Turkey slaughter house, the hydatid cyst was the cause of spleen condemnation (Yibar et al., 2015).

\section{CAUSES OF CONDEMNATIONS OF INTESTINES}

In Alexandria abattoir, the incidence of intestinal lesions was $915(0.72 \%)$ lesions that were significantly higher than those observed in Aswan abattoir 22 (0.09\%). Tuberculosis (0.44\%) and congestions $(0.02 \%)$ were the causes for condemnation of the intestines at Alexandria abattoirs. While the congestion was the only cause for condemnation at Aswan abattoir. However, intestines were free from pathological lesions in Qena abattoir there was no intestinal affection.

Tembo and Nonga (2015) stated that $7.3 \%$ of the examined intestine in Dodoma, Tanzania were commended, the pimply intestine was the main cause of condemnation followed by inflammation of intestinal wall and abscesses formation. While, parasitic infection and abscesses were the main causes of the condemnation of intestine in Ethiopia (Mummed and Webb, 2015).

The findings of the current work showed that there were various diseases related to condemnation of carcasses and organs at the Egyptian slaughterhouse. Among the investigated abattoirs, Aswan had the highest condemnation rate, which can be attributed to the difference in environmental circumstances, including climate temperature, humidity, animal farming and grassland nature. Meanwhile, Shahraki et al. (2018) attributed the difference in the incidence of parasitic infection to racing behavior and environmental circumstances in a study conducted in Iran.

\section{FINANCIAL LOSSES}

An additional aim of the current work was to determine the economic losses due to meat condemnation in the three Egyptian abattoirs. The financial losses in each abattoir were calculated during 2017 - 2018 (Table 5).
The major financial loss was caused by fasciolosis, followed by icterus, pulmonary congestion, the parasitic infection of lung and heart, pulmonary tuberculosis, liver abscesses, tuberculosis affections of quarters. The total two years duration economic loss due to meat condemnation at three abattoirs was estimated as 4529010 Egyptian pound (383063 USD).

A few works have investigated the financial analysis of the disease meat-producing animals in Egypt. In a work performed by Ahmed et al. (2013), 36480 Egyptian pound was lost owing to the condemnation of organs of slaughtered cattle in Ismailia city. The financial loss in the current works was higher than the findings reported (Cadmus and Adesokan, 2009) in Nigeria, which showed a yearly economic loss of 110968 USD. Economic loss due to meat condemnation has been stated by some studies in many countries (Kebede et al., 2011; Regassa et al., 2013). These works stated that parasitic and bacterial diseases could lead to excessive financial loss as well as can harm public health. The major cause of meat condemnation at two slaughter houses in Turkey were hydatidosis, fasciolosis, tuberculosis and icterus and their direct economic loss due to organ condemnation was 16.363 USD (Yibar et al., 2015). A slaughterhouse survey was conducted in Fako slaughterhouse, Cameroon recorded that 357 bovine organs out of 1472 were condemned due to parasitic infection with a related economic loss 2505 USD (Kouam et al., 2019). Kere et al. (2019) revealed that livers and lungs were the most condemned organ in Kenya as a consequence of hydatid cyst infection with an associated financial loss 152003 USD per year. The alteration in the financial loss between various researches could be attributed to the difference in animal population, prices, and incidence of diseases.

\section{CONCLUSION}

Finally, a large amount of money was lost owing to pathological conditions recognized in Egyptian slaughterhouses. This work revealed that tuberculosis, parasitic infection and icterus were the most prevalent affections that had the greatest influence on the Egyptian budget. It is obvious that an adequate preventive and regular application of anti-parasitic drugs and monitoring procedures are needed in Egypt particularly in Aswan province. Appropriate meat examination and hygienic discarding of condemned meat are necessary to secure the human health.

Considerations should be given the affections commonly detected at slaughterhouses. Monitoring programs at the farm should be followed to minimize the hazard 
of infectious diseases and to decrease the financial losses. Moreover, the building of efficiently equipped slaughterhouses and educating of slaughterhouse personnel are critical.

\section{ACKNOWLEDGEMENT}

The authors are grateful to Veterinarians Azza Mofid ElQt, Ahmed Hussein and Mahmoud Fathi directorate of Veterinary Medicine in Alexandria, Qena and Aswan, respectively for their help during data gathering.

\section{CONFLICT OF INTERESTS}

None.

\section{AUTHORS CONTRIBUTION}

Eman Sayed Mohammed and Mohamed Abdelfattah Maky planned the study and gathered the data. Mohamed Abdelfattah Maky discussed the data and drafted the paper.

\section{ETHICAL CODE}

The codes of Alexandria, Qena and Aswan abattoirs were C/0206010312, 270101010617, 580101010310, respectively.

\section{REFERENCES}

-Abdulhameed MF, Habib I, Al-Azizz, SA, Robertson I (2018). Cystic echinococcosis in marketed offal of sheep in Basrah, Iraq: abattoir-based survey and a probabilistic model estimation of the direct economic losses due to hydatid cyst. Parasite Epidemiol. Contl. 3 (1): 43-51. https://doi. org/10.1016/j.parepi.2018.02.002

-Ahmed AM, Ismail SAS, Dessouki AA (2013). Pathological lesions survey and economic loss for male cattle slaughtered at Ismailia abattoir. Int. Food Res. J. 20(2): 857-863.

- Alton GD, Pearl DL, Bateman KG, McNab WB, Berke, O (2010). Factors associated with whole condemnation rates in provincially inspected abattoir in Ontario 2001-2007: Implication for food animal syndromic surveillance. BMC Vet. Res. 6: 42. https://doi.org/10.1186/1746-6148-6-42

- Aminzare M, Hashemi M, Faz SY, Raeisi M, Hassanzadazar H (2018). Prevalence of liver flukes infections and hydatidosis in slaughtered sheep and goats in Nishapour, Khorasan Razavi, Iran. Vet. World. 11(2): 146-150. https://doi. org/10.14202/vetworld.2018.146-150

-Azami M, Anvarinejad M, Ezatpour B, Alirezaei M (2013). Prevalence of hydatidosis in slaughtered animals in Iran. Turk. J. Parasitol. 37: 102-106. https://doi.org/10.5152/ tpd.2013.24

-Beyene T, Hiko A (2019). Zoonotic metacestodes and associated financial loss from cattle slaughtered at Yabello municipal abattoir, Borana-Oromia, Ethiopia. Parasite Epidemiol. Contr. 3: e00096. https://doi.org/10.1016/j.parepi.2019. e00096

-Bin Kabir MH, Eliyas M, Hashem MA, Mohiuddin, Miazi OF (2010). Prevalence of zoonotic parasitic diseases of domestic animals in different abattoir of Comilla and Brahman Baria region in Bangladesh. Univ. J. zool. Rajshahi Univ. 28: 2125. https://doi.org/10.3329/ujzru.v28i0.5281

-Cadmus SIB, Adesokan HK (2009). Causes and implications of bovine organs/offal condemnations in some abattoirs in Western Nigeria. Trop. Anim. Health Pro. 41: 1455-1463. https://doi.org/10.1007/s11250-009-9334-7

-Fekadu A, Legesse E, Tesfaye D (2012). The cause, rate and economic implication of organ condemnation of cattle slaughtered at Jimma municipal abattoir, Southwestern Ethiopia. Glob. Vet. 9(4): 396-400.

-FAO (1994). Food and Agricultural Originations (FAO). Manual for meat inspection for developing countries, FAO. 1994. Rome Italy.

- Fromsa A, Jobre Y (2012). Estimated annual economic loss from organ condemnation, decreased carcass weight and milk yield due to bovine hydatidosis (Echinococcus granulosus, Batsch, 1786) in Ethiopia. Ethiop. Vet. J. 16: 1-14. https:// doi.org/10.4314/evj.v16i2.1

- Ghasemian O, Hoseini G, Soleimani M, Mahmoudi R, Kaboudari A (2018). The prevalence study of hydatid cyst in domesticated slaughtered animals in industrial abattoirs in Iran. J. Bacteriol. Mycol. 6(2): 96-100.

- Gracey JF, Collins DS, Huey RJ (1999). Meat Hygiene.10 th ed., W.B. Saunders Co. 1999. Ltd., London.

-Jaja IF,Mushonga B, Green E,MuchenjeV (2017).A quantitative assessment of causes of bovine liver condemnation and its implication for food security in the eastern cape province South Africa. Sustainability. 9: 736-749. https://doi. org/10.3390/su9050736

-Jibat T, Ejeta G, Asfaw Y, Wudie A (2008). Causes of abattoir condemnation in apparently healthy slaughtered sheep and goats at Helmex abattoir, Debre Zeit, Ethiopia. Rev. Méd. Vét. 159( 5): 305-311.

-Kambarage DM, Kimera SI, Kazwala RR, Mafwere BM (1995). Disease conditions responsible for condemnation of carcasses and organs in short-horn Zebu cattle slaughtered in Tanzania. Prev. Vet. Med. 22: 249-255. https://doi. org/10.1016/0167-5877(94)00421-E

-Kebede N, Gebre-Egziabher Z, Tilahun G, Wossene A (2011). Prevalence and financial effects of hydatidosis in cattle slaughtered in Birre-Sheleko and Dangila abattoirs, Northwestern Ethiopia. Zoonoses Public. Hlth. 58(1): 4146. https://doi.org/10.1111/j.1863-2378.2009.01250.x

-Kere OJ, Joseph E, Jessika BL, Maina KJ (2019). Prevalence and monetary loss due to cystic Echinococcosis in slaughter house livestock: A case study of Migori County, Kenya. Parasite Epidemiol. Contr. 3:105- 113. https://doi.org/10.1016/j. parepi.2019.e00105

- Khanjari A, Partovi R, Abbaszadeh A, Nemati G, Bahonar A, Misaghi A, Basti A, Ilanjegh A, Motaghifar A (2010). A Retrospective Survey of Fasciolosis and Dicrocoeliosis in Slaughtered Animals in Meisam Abattoir, Tehran, Iran (2005-2008) Slaughtered Vet. Res. Forum. 1: 174-178.

-Kouam MK, Meningue R, Fon DE (2019). Parasitic causes of organ condemnation in cattle slaughtered in Fako abattoirs, South-West region of Cameroon, and estimate of 
financial losses. J. Helminthol. 93(3) :367-371. https://doi. org/10.1017/S0022149X18000391

- Maxwell O (2005). Pathological conditions of condemned bovine lungs from abattoirs in Akwa Ibom state, Nigeria. Anim. Res. Int. 2(2): $314-314$.

- Mellau BL, Nonga HE, Karimuribo ED (2011). Slaughter stock abattoir survey of carcasses and organ/offal condemnations in Arusha region, Northern Tanzania. Trop. Anim. Health Pro. 43(4): 857-864. https://doi.org/10.1007/s11250-0109773-1

- MummedYY,Webb EC (2015). Causes of beef carcass and organ condemnations in Ethiopia. Asian J. Anim. Vet. Adv. 10 (4): 147 - 160. https://doi.org/10.3923/ajava.2015.147.160

- Nzalawahe J, Komba EVG (2013). Occurrence and seasonal predisposition of fasciolosis in cattle and goats slaughtered in Kasulu District abattoir, Western Tanzania. R. O. A. V. S3(11): 395-400.

-Pezeshki A, Aminfar H, Aminzare M (2018). An analysis of common foodborne parasitic zoonoses in slaughtered sheep and cattle in Tehran, Iran, during 2015-2018. Vet. World. 11(10) 1486-1490. https://doi.org/10.14202/ vetworld.2018.1486-1490

- Raji MA, Salami S, Ameh JA (2010). Pathological conditions and lesions observed in slaughtered cattle in Zaria abattoir. J. Clin. Pathol. Forensic Med. 1(2): 9 - 12.

- Regassa A, Moje N, Megersa B, Beyene D, Sheferaw D, Debela, E, Abunna F, Skjerve E (2013). Major causes of organs and carcass condemnation in small ruminants slaughtered at Luna Export abattoir, Oromia Regional State, Ethiopia. Prev. Vet. Med. 110(2):139-148. https://doi.org/10.1016/j. prevetmed.2012.11.020

-Shahraki MK, Ghanbarzehi A,Dabirzadeh M (2018). Prevalence and histopathology of Sarcocystosis in slaughtered carcasses in southeast Iran. J. Adv. Vet. Anim. Res. 5(4): 381-387. https://doi.org/10.5455/javar.2018.e288

- Steinfeld H, Wassenaar T, Jutzi, S (2006). Livestock production systems in developing countries: status, drivers, trends. Rev. Sci. Tech. 25: 505-516. https://doi.org/10.20506/ rst.25.2.1677

-Tavassoly, A (2003). Pathological study of renal lesions in the condemned bovine kidneys in Ziaran Slaughter-house. Arch. Razi. Inst. 55(1): 97-104.

- Tembo W, Nonga HE (2015). A survey of the causes of cattle organs and/ or carcass condemnation, financial losses and magnitude of foetal wastage at an abattoir in Dodoma, Tanzania. Onderstepoort J. Vet. 82(1): 7. https://doi. org/10.4102/ojvr.v82i1.855

- Yibar A, Selcuk O, Senlik, B (2015). Major causes of organ/ carcass condemnation and financial loss estimation in animals slaughtered at two abattoirs in Bursa Province, Turkey. Prev. Vet. Med. 118 (1): 28-35. https://doi. org/10.1016/j.prevetmed.2014.11.012

-Youssef AI, Uga S (2014). Review of Parasitic Zoonoses in Egypt. Trop. Med. Health. 42(1): 3-14. https://doi. org/10.2149/tmh.2013-23 